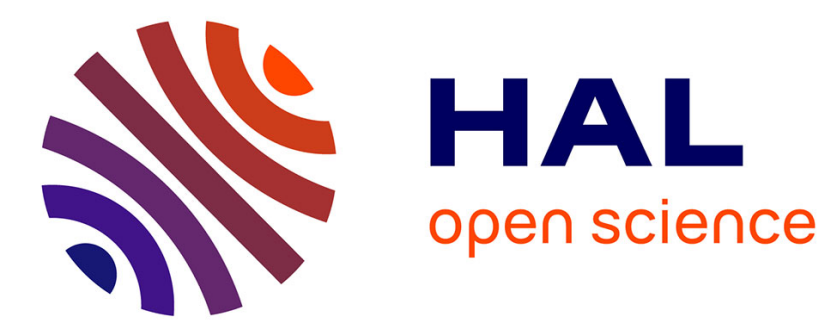

\title{
Lyapunov-based synchronization of networked systems: From continuous-time to hybrid dynamics
}

\author{
Mohamed Maghenem, Romain Postoyan, Antonio Loria, Elena Panteley
}

\section{To cite this version:}

Mohamed Maghenem, Romain Postoyan, Antonio Loria, Elena Panteley. Lyapunov-based synchronization of networked systems: From continuous-time to hybrid dynamics. Annual Reviews in Control, 2020, 50, pp.335-342. 10.1016/j.arcontrol.2020.06.003 . hal-02901359

\section{HAL Id: hal-02901359 \\ https://hal.science/hal-02901359}

Submitted on 17 Jul 2020

HAL is a multi-disciplinary open access archive for the deposit and dissemination of scientific research documents, whether they are published or not. The documents may come from teaching and research institutions in France or abroad, or from public or private research centers.
L'archive ouverte pluridisciplinaire HAL, est destinée au dépôt et à la diffusion de documents scientifiques de niveau recherche, publiés ou non, émanant des établissements d'enseignement et de recherche français ou étrangers, des laboratoires publics ou privés. 


\title{
Lyapunov-Based Synchronization of Networked Systems: From Continuous-Time to Hybrid Dynamics
}

\author{
Mohamed Maghenem ${ }^{\mathrm{a}}$, Romain Postoyan ${ }^{\mathrm{b}}$, Antonio Loría ${ }^{\mathrm{c}}$, Elena Panteley ${ }^{\mathrm{c}, \mathrm{d}}$ \\ ${ }^{a}$ Department of Electrical 8 Computer Engineering, University of California Santa Cruz. California, USA. \\ ${ }^{b}$ Université de Lorraine, CNRS, CRAN, F-54000 Nancy, France. \\ ${ }^{c}$ Laboratoire des Signaux et Systèmes, CNRS, Gif-sur-Yvette, France. \\ ${ }^{d}$ ITMO University, Kronverkskiy av. 49, Saint Petersburg, 197101, Russia.
}

\begin{abstract}
Synchronization pertains to the property of interconnected systems according to which their dynamic behavior is coordinated in an appropriate sense. That is to say, some of their state variables, or functions of the latter for that matter, converge to each other. Synchronization may occur naturally or may be induced, controlled, and it may be present between two systems or among a large number. In the latter case, it is convenient to speak of a network of interconnected systems. Understanding synchronization, and how to control it, is an important paradigm as it is present in a variety of scenarios. These involve, e.g., networks of technological systems (robots and vehicles of different kinds), social networks (by which people exchange opinions and agree, or not), networks of biological systems (uni or pluricellular), etc. Owing to the context, the mathematical models to describe such networks and to define synchronization formally, varies dramatically. Ordinary continuous-time or discrete-time models for which modern control theory and Lyapunov stability theory are tailored result inappropriate to incorporate hybrid phenomena that intervene in the network. These may stem from sudden topology changes, the use of digital or intermittent control strategies, the presence of impacts in the intrinsic dynamics of the nodes, etc. In this invited paper, we give an overview of synchronization control problems, mostly of cooperative control of networks of autonomous vehicles (based on continuous-time models). For that matter, the first part of the paper focuses on the main contributions of [1]. Then, we give further perspectives on what we consider significant open problems on synchronization of hybrid systems and hybrid networks.
\end{abstract}

Keywords: Control engineering; Synchronization; Networks of dynamical systems; Safety; Hybrid dynamical systems; Lyapunov methods.

\section{Introduction}

Generally speaking, for a network of dynamical systems, inducing synchronization consists in designing a controller (often one for each node in the network) so that an output or a state variable of each system acquires, asymptotically, a common behavior. For example, the spatial positions of network-interconnected autonomous vehicles (such as drones, mobile robots, platoons of cars, etc.) may be controlled to form a specified geometric pattern, a formation of sorts; the currents and voltages in a network of DC-AC inverters may be controlled to reach the same frequency of oscillation.

Depending on the nature of interaction among the nodes in the network, we distinguish between centralized and decentralized control schemes. In the first case, each node receives global information dictating its desired behavior. This may consist in a desired set-point (a specific position or oscillating frequency), a reference

Email address: mmaghene@ucsc.edu (Mohamed Maghenem) trajectory, or a desired dynamical behavior in more complex cases. In centralized control approaches, each node has an independent task to ensure, which is defined by a central entity. These approaches are often used when the network is composed of a relatively small number of nodes, but abundant computation and communication capacities may be required for the control entity. Decentralized approaches, as we consider in this article, overcome these limitations. There, the control input for each node depends only on local information provided by a given set of nodes called neighbors. The network topology, which determines how the information among the neighbors flows, is typically defined using a communication graph [1]. The communication graph plays a key role in the analysis and design of networked systems.

Decentralized synchronization in networked systems is an active and challenging research topic that has continuously attracted undivided attention from many researchers over the previous two decades - see, for instance, [1 4] in which both continuous and discrete-time networks are considered. Such is the case in engineering 
disciplines where technological solutions involve groups of systems required to cooperate to complete a common task. Some common examples of these are related, but are not limited, to robotic manipulation and the use of autonomous vehicles [5], but they are also common in energy networks [10, for instance. Other disciplines include neuroscience, in which networks and sub-networks (clusters) formed due to neuronal population activities and interactions are studied 11, social science, in which a subject of study is that of opinion networks formed due to the interactions among individuals [12, and geography, in which groups of populations that interact when reacting to catastrophes are studied 13. While the systems models involved in each of these instances are different, a common denominator is the presence of a network composed of nodes having a dynamic behaviors that interact locally with the aim at reaching a common global goal. From a systems-theory viewpoint, at least two primary challenges can be identified when contributing to synchronization problems. The first one arises from recognizing that communication among the nodes is subject to constraints; this includes scenarios where the transfer of information is unreliable, time-dependent, or subjected to delays. The second challenge stems from considering the complexity of the nodes' dynamics; these may be linear, nonlinear or hybrid in nature and, moreover, may vary throughout the network - such is the case of heterogeneous networks.

Some of these aspects have been addressed in [14, in scenarios pertaining to electromechanical engineering and robotics. The main originality of [14 is to propose advanced Lyapunov-based approaches inspired by techniques for stand-alone nonlinear time-varying systems to analyze the coordination problems after transforming it into global stabilization of a closed, but unbounded, set. Then, Lyapunov-based techniques, via the construction of strict and differentiable Lyapunov functions, are proposed for the network's model after interconnection. This allows to derive several systematic methods to analyze the coordination task, the network's performance, and its robustness with respect to perturbations and delays affecting the transfer of information among the nodes. Note that, in related literature, the coordination task is usually analyzed via trajectory-based approaches or using weak Lyapunov functions, in the sense that the Lyapunov function does not systematically allow the verification the coordination task. In that way, for autonomous mobile robots, different coordination tasks are studied in [14, such as consensus and trajectory-tracking formation control or, for networks of oscillators, the frequency synchronization problem has been addressed. In regards to the nature of interaction and transfer of information among the nodes, several scenarios are considered; these include time-varying interconnections as well as communication delays. As far as the intrinsic dynamics of the nodes is considered, only some continuous-time models, such as single and double integrators, time-varying planar oscillators, and nonholonomic unicycles, have been considered.

The results contained in 14 concern exclusively interconnected systems described by continuous-time models. However, the problems solved therein lead to more general open questions that transcend these systems and are, in our opinion, of sufficient significance to awake the interest of a wide readership. The problems that we describe are related to the study of synchronization problems described previously, but in the context of hybrid networked systems. In particular, it is of major importance to handle the presence of discrete phenomena that are coupled with the continuous-time dynamics of the nodes that compose the network. A prominent example that makes this problem relevant is that of digital or intermittent control strategies that interact with continuous-time models, which captures constrained control actions as in power networks [15, 16, in valve-based control systems [17, or, in networked control systems [18, 19]. It also captures the creation (resp. loss) of links and the addition (resp. removal) of nodes as in social networks 20 22]. Furthermore, in the intrinsic behavior of the nodes, one must also consider systems affected by discontinuities such as impacts and instantaneous jumps like in mechanical systems with impacts 23, 24] or in cyber-physical systems [25 27]. Hence, such research aims at covering unexplored aspects which, nonetheless, are naturally motivated by intrinsic characteristics of modern systems networks.

The rest of this paper is organized as follows. In the next section, we describe the main contributions of 14 . In section 3 , we discuss the relevance and challenges related to synchronization in hybrid networks. Finally, in Section 4. we provide some research directions.

\section{Synchronization in Continuous-Time Net- worked Systems}

\subsection{Main Idea}

In [14, original Lyapunov-based approaches are developed to analyze networks where the nodes are interconnected via decentralized control laws. More precisely, the proposed approaches consist in transforming the coordination task into global stabilization of a closed set. The analysis of the coordination task has been conducted using Lyapunov's direct method. That is, original constructions of strict and differentiable Lyapunov functions, for classes of nonlinear time-varying systems modeling the network after interconnection, are provided. Compared to related literature, where the coordination task is usually analyzed using trajectory-based approaches or using weak Lyapunov functions, in the sense that the Lyapunov function does not systematically allow the verification the coordination 
task, the solutions proposed in 14 allow a systematic analysis of the coordination task as well as the network's performance, e.g., in terms of convergence rates. Moreover, a systematic robustness analysis with respect to perturbations and delays affecting the transfer of information among the nodes is also carried out. Thanks to these approaches, various important coordination problems have been solved in 14. These problems have been solved for different graph topologies, depending on how the information flows among the nodes, and for diverse target trajectories.

\subsection{Leader-Follower Formation Control for Nonholo- nomic Vehicles}

In leader-follower formation problems for mobile vehicles, the coordination task consists in making the vehicles maintain a given geometric posture in terms of both, positions and orientations, relative to that of a leader and track its trajectory 28]. For a network of nonholonomic vehicles and due to the nonholonomic restriction, solving this problem under a general leader's trajectory, using a unique continuous feeedback control law, is a challenging problem, especially to establish uniform asymptotic stability. Indeed, it is shown in 29 that nonholonomic systems are not asymptotically stabilizable to arbitrary leaders trajectories having piecewise continuous velocities via continuous controllers, not even if the controllers are time-varying (the possibility of using discontinuous controllers is not analyzed in 29 and we are not aware of any other work for that matter). In the literature, for the simpler one-leader-and-one-follower case, the tracking problem has been studied in e.g., 30 34 under particular scenarios of the leader's trajectories. To the best of our knowledge, the only article that allows for general leader's trajectories and for a network of nonholonomic vehicles is [28]. The control design method in the latter reference follows the framework proposed in 35. However, it is only established that the formation errors converge to an arbitrarily small compact ball centered at the origin.

In [14, see also the associated publications [34, 3639, decentralized feedback control laws are proposed to solve the leader-follower formation control problem for a network of nonholonomic mobile robots. The interconnections among the nodes is assumed to be a directed spanning-tree topology; namely, each node has only one leader and can have multiple followers. The formation pattern is specified in terms of both the positions and the orientations. The network of vehicles is shown to asymptotically reach the desired geometric pattern around the leader and to track the leader's trajectory. Although the communication graph is simple, the originality of this work consists in providing strong stability guarantees; namely, global uniform asymptotic stability, for general leader's trajectories and using continuous-time feedback laws. That is, the leader's trajectory is allowed to con- verge to a static location (parking problem), and can also have a general time-varying velocity profile.

\subsection{Leaderless Formation Control for Nonholonomic Ve- hicles}

In the context of the previously described problems, the network is assumed to contain a leader. However, in some applications, the formation does not have a leader. Hence, the coordination task consists in making the vehicles maintain a given geometric posture, in terms of positions and orientations, around a center point. This problem has been studied for a network of nonholonomic vehicles in [40, 44] using decentralized continuous control laws. In the aforementioned references, it is shown that the nodes converge asymptotically to the required formation. However, the stability properties of the formation errors have not been analyzed. While ensuring convergence to the desired formation is important, it is also essential to have guarantees about the convergence times, in particular that two nearby initial conditions have approximately to the same convergence rate, as well as to make sure that when the robots are "close" to form the formation, they preserve this property for all times. One way to capture these features is to ensure that uniform global asymptotic stability properties are satisfied.

The leaderless formation control problem for a network of nonholonomic mobile vehicles is studied in [14, see also 45 47. The communication graph, in this case, is assumed to be a general bidirectional graph. A continuous and time-varying decentralized interconnection algorithm is proposed and the network after interconnection is showed to converge to the desired formation in terms of both positions and orientations. A significant contribution consists in establishing uniform global asymptotic stability of the formation error for the closed-loop network. Furthermore, in contrast to most of the previous-cited works, where only the kinematics model is considered, we use a model augmented by two integrators, which represent the feedback-linearized velocity dynamics. Furthermore, original tools, via the construction of strict Lyapunov functions, are proposed to analyze stability of the network in closed loop. Using these tools, in [46, 47, we allowed the transfer of information among the nodes to be affected by general time-varying delays. The same approach was used in 44 for a network of Euler-Lagrange systems.

\subsection{Synchronization in Heterogeneous Networks Using Strong Interconnections}

In general, the intrinsic dynamics of the nodes are not necessarily the same. Such is the case, for example, of networks of oscillators, in which the intrinsic frequency of the nodes is not identical [48. We refer to such networks as heterogenous. For a network of nonlinear systems, in which all the nodes are identical and the interconnection gain is sufficiently high, it is expected that the nodes synchronize; 
namely, that the state variables reach a common value. Furthermore, it is intuitively clear that a synchronized behavior of such a network corresponds, roughly speaking, to all the nodes adopting the motion of a single isolated unit [4]. However, in the case of heterogeneous networks, the paradigm of asymptotic synchronization is much more complex: the existence of a synchronization manifold is not guaranteed 50. Hence, in lieu of adopting the behavior of one of the nodes, some type of internal model is necessary and sufficient for synchronization [50] and, in general, only "practical" synchronization is usually ensured in the literature considering heterogeneous networks [51 56. In [53, 54 a novel framework of analysis of heterogeneous networks was developed upon the premise that the collective behavior of network-interconnected systems is dichotomic: it consists, on the one hand, in a "weighted averaged" motion determined by the so-called emergent dynamics and, on the other, in the dynamics of the synchronization errors of each individual unit in the network, relatively to the collective behavior. Then, we say that the network synchronizes or, more precisely, achieves dynamic consensus if all the nodes adopt the emergent behavior in steady state.

This approach was pursued and extended in 57, 58 ${ }^{1}$ where it is assumed that the emergent dynamics admits a limit cycle with certain stability properties. It is shown that each individual interconnected node also admits a limit cycle with the same frequency and the same stability properties as the one in the emergent dynamics. The different nodes are interconnected via a general directed graph. For the first time in the literature of synchronization, the analysis was based on singular-perturbation theory. Indeed, due to the strong interconnection, it is showed that the emergent dynamics corresponds to a slow system while the dynamics of the synchronization errors form a fast one.

\subsection{Consensus Under Time-Varying Graphs}

A last contribution of 14 is dedicated to the consensus problem where the graph is time-varying. Considering time-varying graphs captures different scenarios in which the transfer of information among the nodes is unreliable, the information flow through a restricted amount of channels, or, when the nodes have a limited sensing range. Consensus problem under time-varying and switching graphs has been widely studied in the literature, see e.g., [3, 59 65], mostly for nodes with single or double integrator dynamics, under different graph-connectivity conditions. The approach in the aforementioned references uses non-systematic trajectory-based approaches via non-smooth Lyapunov functions, which makes the analysis of robustness challenging.

\footnotetext{
${ }^{1}$ The contributions in [57, 58 have not been included in 14 and 58. will be submitted soon.
}

In $66 \sqrt{68}{ }^{2}$ a novel analysis approach for time-varying consensus protocols employing the notions of persistency of excitation and strict Lyapunov functions are provided. Those approaches rely on stability theory of so-called gradient-descent and model-reference-adaptivecontrol systems, known in adaptive control theory [69]. That is, the classical consensus paradigm is recast into a problem of stability analysis for systems with persistency of excitation. The analysis relies on the edgeagreement transformation based on the relationship between node and edge-agreement protocol 70 . The interconnection graph is assumed time-varying while maintaining a mild persistent connectivity among the nodes. The time-varying interconnections, individually, are assumed to be persistently exciting. Furthermore, networks composed of single-integrator nodes, double-integrator nodes, and time-varying planar oscillators are studied. Finally, different convergence rate estimates, for the consensus error coordinates, are provided.

\section{Towards Hybrid Networked Systems}

All the results mentioned in Section 2 concentrate on networked systems with continuous-time dynamics. It appears that in many situations, the overall system also exhibits discontinuous/jump dynamics either at the nodes' level or at the network's level. Before describing hybrid networks, in the next section, hybrid dynamical systems are introduced according to the modeling framework of 71].

\subsection{Hybrid Dynamical Systems}

In [71, a hybrid dynamical system is seen as the following combination of a constrained differential equation and a constrained difference equation

$$
\begin{cases}(x, u) \in C & \dot{x}=f(x, u) \\ (x, u) \in D & x^{+}=g(x, u) .\end{cases}
$$

The state of the hybrid system, represented by $x$, can change according to the differential equation $\dot{x}=f(x, u)$ while in the flow set $C$, and it can change according to a difference equation $x^{+}=g(x, u)$ while in the jump set $D$. When $x \in C \cap D$, the solution can either jump or flow if flowing keeps the solution in $C$. The notation $\dot{x}$ represents the time derivative of the state $x$, while $x^{+}$represents the value of the state after an instantaneous jump.

The strength of this formalism resides on the possibility of capturing, in a single representation, heterogeneous dynamics involving both continuous and discrete evolutions. It allows to cover many real-world applications that cannot be modeled precisely as purely continuous-time systems nor as purely discrete-time systems. Finally, it allows

\footnotetext{
${ }^{2}$ The works in $67 \sqrt[68]{68}$ have not been included 14 .
} 
the use and the extension of state-of-the-art tools to analyze continuous and discrete-time systems (stability, robustness, safety, etc). Indeed, a range tools for analysis and control design of systems described by (1) are by now available in the literature [71.

\subsection{Motivation}

Hybrid phenomena at the node's level are common in the case of mechanical systems with impacts, such as walking robots [23, 72, juggling mechanisms [24, 73], and classes of cyber-physical systems [25, 74. Furthermore, hybrid phenomena at the network's level are common in many applications. For example, when interconnecting nodes under constrained control actions, which is the case in power converters within energy grids [15, 16. and in valve-based control systems [17, where the input switches among a finite set of operation modes. In networks of event-triggered and self-triggered control systems [19, 25, 75,77, where the input is updated only at particular events and remains constant between events. Finally, in network control systems [18, 26, 27, where all the data (inputs and measurements) flow through the same channel; hence, the measurements and the control actions are updated only at particular intervals of time.

While there are now numerous approaches to analyze and design continuous-time and discrete-time interconnected systems, there is a need for methodological tools, which are adapted to hybrid phenomena in the literature.

\subsection{Handling Hybrid Phenomena in Networked Systems}

In the literature, fundamental links between algebraic graph theory and synchronization have been established for different scenarios of continuous and discrete-time networked systems. However, the tools that are available to date to analyze synchronization are not always applicable when both continuous and discrete phenomena coappear. Indeed, assume that the interaction among the hybrid nodes occurs only during their continuous-time, or only during their discrete-time evolution. Such is the case for mechanical nodes with impacts 23] or for networks under any event-based or intermittent interconnections [78. In this case, one needs to show that the network's attitude to synchronize during one of the two modes (flowing or jumping) is robust with respect to the other mode along which the interconnection is lost. However, most of the existing techniques to show synchronization for purely continuoustime or purely discrete-time networks are trajectory-based approaches [62, 79] and cannot be easily extended in order to analyze robustness. Another example, where classical tools do not apply, is that of hybrid networks operating in clusters. In this case, sub-networks are formed due to their strong interconnections. The nodes within the same sub-network tend to synchronize quickly (fast dynamics) before interacting with the other sub-networks (slow dynamics) [80 83. The global hybrid network exhibits a multi-time-scale dynamics 84, 85. As discussed in Section 2.4 singular-perturbation theory is useful to analyze synchronization in the presence strong interconnections. However, for stand-alone hybrid singularly perturbed systems and even for a one-system case, only few results are available, see e.g., [86 88 for more discussion.

The main originality of [14 consists in providing a range of advanced Lyapunov-based approaches for setstability, inspired by techniques for nonlinear time-varying systems, for interconnected continuous-time systems. Similar Lyapunov-based tools exist for hybrid systems [71. In particular, a range of tools are available for the analysis of set stability. Hence, there is hope to follow similar approaches when analyzing hybrid networks. For this purpose, several key questions would need to be addressed.

1. The modeling of the interconnections among the nodes, which is not a trivial task and requires special care to preserve the so-called basic conditions for the network in closed loop [89, 90. Hybrid basic conditions are essential for hybrid systems to ensure some robustness properties, see [71.

2. The current approaches to address hybrid interconnected systems consist in modeling the overall network as a hybrid system and to apply related results to conclude about stability like in [25]. For instance, it would be much more natural to derive global properties based on the local properties of each node and the interconnection graph, as suggested in [91.

3. The persistence of excitation property, which have been extensively used in 14, extends to the notions of persistent flow and persistent jump in the hybrid systems [92, which need to be better understood in terms of their implication on synchronization.

4. The possibility of a mismatch between the times when the hybrid nodes jump would considerably increase the difficulty to analyze synchronization. This fact has been mentioned in the particular context of hybrid observers in 93 .

\section{Challenging Problems}

The aforementioned questions are general and fundamental. It therefore seems appropriate to follow a divideand-conquer strategy by considering, separately, situations where the hybrid phenomena are due to the node dynamics, from those where these are due to the control strategy as discussed in the following.

\subsection{Synchronizing Intrinsically Hybrid Nodes}

Networks of hybrid nodes include networks of switched systems (like power converters), mechanical systems with impacts, or cyber-physical systems. Because such networks are challenging, it would be relevant to first concentrate on situations where the interaction among the systems occurs only during their continuous-time evolution, which is the case in walking robots, or only during 
their discrete-time evolution, which is the case of juggling mechanisms. In each of the previously-cited scenarios, we need to provide robustness-analysis tools in order to conclude when the network's attitude to synchronize during one of the two modes of evolution (flowing or jumping) is robust with respect to the other mode, where the interconnections are not present, and also with respect to the mismatch in the jump times as the nodes do not necessarily jump at the same time. The qualitative Lyapunov-based techniques for time-varying systems proposed in [14], as described in Section 2 and 67, 94, is relevant here and would need to be adapted to this context.

\subsection{Synchronization Using Hybrid Controllers}

A node may also be hybrid because of the used local controller. There, a hybrid controller is a hybrid system admitting the system or part of the system's state variables as inputs and generates, as outputs, the system's control inputs. First, this class of controllers can overcome fundamental limitations of continuous-time controllers e.g. 95 97. They also offer the possibility to solve the combined stability-safety control problem robustly [98. More importantly, hybrid controllers offer the flexibility to model different scenarios of constrained interconnection among the nodes [99] and they naturally arise in presence of sampling [25, 27, 27, 78, 89, 100].

A concrete research problem, related to synchronization in networks of non-hybrid nodes using hybrid interconnections, is to analyze networks operating in clusters under event-based interconnections. In this case, the global network may exhibit a multi-time-scale dynamics. Moreover, since the interconnections are intermittent, the resulting closed-loop network exhibits hybrid phenomena. A promising research direction that contribute to this question, consists in extending the singular-perturbation-based framework proposed in 101, 102 as described in Section 2.4. The tools developed in the aforementioned references exploit the continuity of the stability properties of classes of singularly perturbed systems under small perturbations. Showing such a continuity property for classes of hybrid singularly perturbed systems is an open question to the best of our knowledge.

\subsection{Safety Specifications in Hybrid Networks}

Usually, in many real-world applications, safety requirements need to be guaranteed when solving synchronization problems. Safety is also named obstacle avoidance in the particular context of mobile robotics. Safety while synchronizing a network of dynamical systems means that the trajectories of the network in closed loop, when starting from a given set of initial conditions, remain within a safe region of the state space while the variables of interest converge to the agreement. Barrier functions are analogues to Lyapunov functions are have been usually used to guarantee safety in dynamical systems [103, 104. They have been also used for networked systems in [105109. Recently, in 110, barrier functions are used to show safety for hybrid systems in the form of $\mathcal{H}$. However, to the best of our knowledge, establishing distributed safety criteria for hybrid networks has not been done yet.

\section{Conclusion}

This article reviews the main contributions presented in 14] where original Lyapunov-based approaches for set stability have been developed for the synchronization of networked continuous-time systems. The approach of 14 together with the recent advances on the Lyapunov stability theory for hybrid systems allow us to envision a range of exciting perspectives for the synchronization of hybrid interconnected systems. The main motivations of this research direction are discussed and some open problems are proposed.

\section{Acknowledgment}

This work was partially funded by ANR via grant HANDY, number ANR-18-CE40-0010. The first author feels deeply grateful to Prof. Ricardo G. Sanfelice for introducing him to the area of hybrid systems.

\section{References}

[1] W. Ren and R. W. Beard. Distributed consensus in multivehicle cooperative control. Springer verlag, 2005.

[2] J. Tsitsiklis, D. Bertsekas, and M. Athans. Distributed asynchronous deterministic and stochastic gradient optimization algorithms. IEEE Transactions on Automatic Control, 31(9):803-812, 1986.

[3] A. Jadbabaie, J. Lin, and A. S. Morse. Coordination of groups of mobile autonomous agents using nearest neighbor rules. IEEE Trans. on Automatic Control, 48(6):988-1001, 2003.

[4] R. R. Olfati-Saber and R. M. Murray. Consensus problems in networks of agents with switching topology and time-delays. IEEE Trans. on Automatic Control, 49(9):1520-1533, 2004.

[5] A. Rodriguez-Angeles and H. Nijmeijer. Mutual synchronization of robots via estimated state feedback: a cooperative approach. IEEE Transactions on Control Systems Technology, 12(4):542-554, 2004.

[6] A. Dong and J. A. Farrell. Cooperative control of multiple nonholonomic mobile agents. IEEE Trans. on Automatic Control, 53(6):1434-1447, 2008.

[7] Y. Eun and H. Bang. Cooperative control of multiple unmanned aerial vehicles using the potential field theory. Journal of Aircraft, 43(6):1805-1814, 2006.

[8] D. J. W. Belleter and K. Y. Pettersen. Leader-follower synchronisation for a class of underactuated systems. In Nonlinear Systems: Techniques for Dynamical Analysis and Control, pages 157-179. Springer International Publishing, Cham, 2017.

[9] X. Liu, Y. Guo, and P. Lu. Robust attitude coordination control for satellite formation with matched perturbations and measurement noises. In Proceedings of the 2014 American Control Conference, pages 3893-3898, June 2014.

[10] C. De Persis, E. Weitenberg, and F. Dörfler. A power consensus algorithm for dc microgrids. Automatica, 89:364-375, 2018.

[11] F. Wendling, J-J. Bellanger, F. Bartolomei, and P. Chauvel. Relevance of nonlinear lumped-parameter models in the analysis of depth-eeg epileptic signals. Biological Cybernetics, $83(4): 367-378,2000$. 
[12] S. E. Parsegov, A. V. Proskurnikov, R. Tempo, and N. E. Friedkin. A new model of opinion dynamics for social actors with multiple interdependent attitudes and prejudices. In Proceedings of the 2015 54th IEEE Conference on Decision and Control (CDC), pages 3475-3480, Dec 2015.

[13] G. Cantin. Nonidentical coupled networks with a geographical model for human behaviors during catastrophic events. International Journal of Bifurcation and Chaos, 27(14):1750213, 2017.

[14] M. Maghenem. Stability and stabilisation of networked systems. PhD thesis, Université Paris Saclay, Gif sur Yvette, France, 2017.

[15] L. Torquati, R. G. Sanfelice, and L. Zaccarian. A hybrid predictive control algorithm for tracking in a single-phase DC/AC inverter. In Proceedings of the 2017 IEEE Conference on Control Technology and Applications (CCTA), pages 904-909, 2017.

[16] G. Beneux, P. Riedinger, J. Daafouz, and L. Grimaud. Robust stabilization of switched affine systems with unknown parameters and its application to DC/DC flyback converters. In Proceedings of the 2017 American Control Conference (ACC), pages 4528-4533, 2017.

[17] G. Panzani, T. Colombo, S. M. Savaresi, and L. Zacearan. Hybrid control of a hydro-pneumatic tractor suspension. In Proceedings of the 2017 IEEE 56th Annual Conference on Decision and Control (CDC), pages 250-255, 2017.

[18] J. P. Hespanha, P. Naghshtabrizi, and Y. Xu. A survey of recent results in networked control systems. Proceedings of the IEEE, 95(1):138-162, 2007.

[19] R. Postoyan, N. Van de Wouw, D. Nešić, and M. Heemels. Tracking control for nonlinear networked control systems. IEEE Transactions on Automatic Control, 59(6):1539-1554, 2014.

[20] I. Morărescú and A. Girard. Opinion dynamics with decaying confidence: Application to community detection in graphs. IEEE Transactions on Automatic Control, 56(8):1862-1873, 2011.

[21] P. Frasca, S. Tarbouriech, and L. Zaccarian. Hybrid models of opinion dynamics with opinion-dependent connectivity. Automatica, 100:153-161, 2019.

[22] S. Mariano, I. C. Morărescu, R. Postoyan, and L. Zaccarian. A hybrid model of opinion dynamics with memory-based connectivity. IEEE Control Systems Letters, 2020.

[23] E. R. Westervelt, J. W. Grizzle, and D. E. Koditschek. Hybrid zero dynamics of planar biped walkers. IEEE Transactions on Automatic Control, 48(1):42-56, 2003.

[24] S. Jiménez-Leudo, N. Quijano, and C. F. Rodríguez. Juggler system: Hybrid model and implementation. In Proceedings of the ASME 2015 Dynamic Systems and Control Conference. American Society of Mechanical Engineers, 2015.

[25] C. De Persis and R. Postoyan. A Lyapunov redesign of coordination algorithms for cyber-physical systems. IEEE Transactions on Automatic Control, 62(2):808-823, 2017.

[26] D.P. Borgers, R. Geiselhart, and W.P.M.H. Heemels. Tradeoffs between quality-of-control and quality-of-service in large-scale nonlinear networked control systems. Nonlinear Analysis: Hybrid Systems, 23:142 - 165, 2017.

[27] C. Nowzari, E. Garcia, and J. Cortés. Event-triggered communication and control of networked systems for multi-agent consensus. Automatica, 105:1 - 27, 2019.

[28] W. Wang, J. Huang, C. Wen, and H. Fan. Distributed adaptive control for consensus tracking with application to formation control of nonholonomic mobile robots. Automatica, 50(4):1254-1263, 2014.

[29] D. A. Lizárraga. Obstructions to the existence of universal stabilizers for smooth control systems. Mathematics of Control, Signals and Systems, 16:255-277, 2004.

[30] Y. Wang, Z. Miao, H. Zhong, and Q. Pan. Simultaneous stabilization and tracking of nonholonomic mobile robots: A Lyapunov-based approach. IEEE Trans. on Control Systems Technology, 23(4):1440-1450, July 2015.

[31] T. C. Lee, Kai. T. Song, C. H. Lee, and C. C. Teng. Track- ing control of unicycle-modeled mobile robots using a saturation feedback controller. IEEE Trans. Contr. Syst. Technol., 9(2):305-318, Mar 2001.

[32] P. Morin and C. Samson. Control of nonholonomic mobile robots based on the transverse function approach. IEEE Trans. on Robotics, 25(5):1058-1073, 2009.

[33] K. D. Do, Z.-P. Jiang, and J. Pan. Simultaneous tracking and stabilization of mobile robots: an adaptive approach. IEEE Trans. on Automatic Control, 49(7):1147-1152, 2004.

[34] M. Maghenem, A. Loría, and E. Panteley. A unique robust controller for tracking and stabilisation of non-holonomic vehicles. International Journal of Control, pages 1-12, 2018.

[35] P. Morin and C. Samson. Practical stabilization of driftless systems on lie groups: the transverse function approach. IEEE Trans. on Automatic Control, 48(9):1496-1508, 2003.

[36] M. Maghenem, A. Loría, and E. Panteley. A cascades approach to formation-tracking stabilization of force-controlled autonomous vehicles. IEEE Transactions on Automatic Control, 63(8):2662-2669, 2017.

[37] M. Maghenem, A. Loría, and E. Panteley. A robust $\delta$ persistently exciting controller for leader-follower trackingagreement of multiple vehicles. European Journal of Control, 40:1-12, 2018. Appeared online: sept. 2017. DOI: 10.1016/j.ejcon.2017.09.001.

[38] M. Maghenem, A. Loría, and E. Panteley. Formation-tracking control of autonomous vehicles under relaxed persistency of excitation conditions. IEEE Transactions on Control Systems Technology, 26(5):1860-1865, 2018.

[39] M. A. Maghenem, A. Loría, and E. Panteley. Cascades-based leader-follower formation-tracking and stabilization of multiple nonholonomic vehicles. IEEE Transactions on Automatic Control, 2019.

[40] Z. Peng, G. Wen, A. Rahmani, and Y. Yu. Distributed consensus-based formation control for multiple nonholonomic mobile robots with a specified reference trajectory. International Journal of Systems Science, 46(8):1447-1457, 2015.

[41] C. Yang, W. Xie, C. Lei, and B. Ma. Smooth time-varying formation control of multiple nonholonomic agents. In Proc. of the 2015 Chinese Intelligent Systems Conference, pages 283291. Springer, 2016.

[42] W. Dong and J. A. Farrell. Consensus of multiple nonholonomic systems. In Proc. of the 47th IEEE Conf. on Decision and Control, pages 2270-2275. IEEE, 2008.

[43] A. Ajorlou, M. M. Asadi, A. G. Aghdam, and S. Blouin. Distributed consensus control of unicycle agents in the presence of external disturbances. Systems \&6 Control Letters, 82:86-90, 2015.

[44] E. Nuño, I. Sarras, A. Loría, M. Maghenem, E. Cruz-Zavala, and E. Panteley. Strict Lyapunov-Krasovskii functionals for undirected networks of Euler-Lagrange systems with timevarying delays. Systems \& Control Letters, 135:104579, 2020.

[45] M. Maghenem, A. Bautista-Castillo, E. Nuño, A. Loría, and E. Panteley. Consensus of multi-agent systems with nonholonomic restrictions via Lyapunovs direct method. IEEE Control Systems Letters (L-CSS), 3(2):344-349, 2018.

[46] M. Maghenem, E. Nuño, A. Loría, and E. Panteley. Partialconsensus control of nonholonomic vehicles with delayed local measurements. To appear in IEEE Trans. on Automatic Control, 2020.

[47] M. Maghenem, E. Nuño, A. Loría, and E. Panteley. Distributed full-consensus control of nonholonomic vehicles under non-differentiable measurement delays. To appear in IEEE Control Systems Letters (L-CSS), 2020.

[48] D. Wilson, S. Faramarzi, J. Moehlis, M. R. Tinsley, and K. Showalter. Synchronization of heterogeneous oscillator populations in response to weak and strong coupling. Chaos: An Interdisciplinary Journal of Nonlinear Science, 28(12):123114, 2018.

[49] Z. Aminzare, B. Dey, E. N. Davison, and N. E. Leonard. Cluster synchronization of diffusively coupled nonlinear systems: A contraction-based approach. Journal of Nonlinear Science, 
pages $1-23,2018$.

[50] P. Wieland, R. Sepulchre, and F. Allgöwer. An internal model principle is necessary and sufficient for linear output synchronization. Automatica, 47(5):1068-1074, 2011.

[51] J. M. Montenbruck, M. Bürger, and F. Allgöwer. Practical synchronization with diffusive couplings. Automatica, 53:235$243,2015$.

[52] T. Liu, D. Hill, and J. Zhao. Output synchronization of dynamical networks with incrementally-dissipative nodes and switching topology. IEEE Trans. on Circ. Syst. I: Fundamental Theory and Applications, 62(9):2312-2323, 2015.

[53] E. Panteley. A stability-theory perspective to synchronisation of heterogeneous networks. Habilitation à diriger des recherches (DrSc dissertation). Université Paris Sud, Orsay, France, 2015.

[54] E. Panteley and A. Loría. Synchronization and dynamic consensus of heterogeneous networked systems. IEEE Trans. on Automatic Control, 62(8):3758-3773, 2017.

[55] J. G. Lee and H. Shim. A tool for analysis and synthesis of heterogeneous multi-agent systems under rank-deficient coupling. Automatica, 117:108952, 2020.

[56] E. Panteley, A. Loría, and A. El-Ati. Practical dynamic consensus of stuartlandau oscillators over heterogeneous networks. International Journal of Control, 93(2):261-273, 2020.

[57] M. Maghenem, E. Panteley, and A. Loría. Singularperturbations-based analysis of synchronization in heterogeneous networks: A case-study. In Proceedings of the 55th IEEE Conf. Decision and Control, pages 2581-2586, Las Vegas, NV, USA, 2016

[58] M. Maghenem, E. Panteley, and A. Loría. Singularperturbations-based analysis of dynamic consensus in heterogeneous networks. Technical report, CNRS, CentraleSupelec, 2017. Technical report in progress; to be submitted to IEEE Trans. on Automatic Control.

[59] A. R. Teel, A. Loría, E. Panteley, and D. Popović. Smooth time-varying stabilization of driftless systems over communication channels. Systems \& Control Letters, 55(12):982 - 991, 2006.

[60] L. Moreau. Stability of continuous-time distributed consensus algorithms. In Proc. of the 43rd. IEEE Conf. on Decision and Control, volume 4, pages 3998-4003, 2004

[61] W. Ren. Synchronization of coupled harmonic oscillators with local interaction. Automatica, 44(12):3195-3200, 2008.

[62] S. Martin and A. Girard. Continuous-time consensus under persistent connectivity and slow divergence of reciprocal interaction weights. SIAM Journal on Control and Optimization, 51(3):2568-2584, 2013

[63] B. D. O. Anderson, G. Shi, and J. Trumpf. Convergence and state reconstruction of time-varying multi-agent systems from complete observability theory. IEEE Trans. on Automatic Control, 62(5):2519-2523, 2017.

[64] N. Alvarez-Jarquin, A. Loría, and J. L. Avila. Consensus under switching spanning-tree topologies and persistently exciting interconnections. In 2018 Annual American Control Conference $(A C C)$, pages 4578-4583, 2018.

[65] R. Goebel and R. G. Sanfelice. A unifying convex analysis and switching system approach to consensus with undirected communication graphs. Automatica, 111:108598, 2020.

[66] M. Maghenem and A. Loría. Lyapunov functions for persistently-excited cascaded time-varying systems: Application in consensus analysis. IEEE Trans. on Automatic Control, 62(7):3416-3422, 2017.

[67] N. R. Chowdhury, S. Sukumar, M. Maghenem, and A. Loria. On the estimation of algebraic connectivity in graphs with persistently exciting interconnections. Int. J. of Contr., 91(1):132-144, 2018.

[68] M. Maghenem, H. Lekoufouet, A. Loría, and E. Panteley. Decentralized synchronization of time-varying oscillators under time-varying bidirectional graphs. In Proceedings of the 2019 American Control Conference (ACC), pages 4018-4023, July 2019 .

[69] K. S. Narendra and A. M. Annaswamy. Persistent excitation in adaptive systems. Int. J. of Contr., 45(1):127-160, 1987.

[70] M. Mesbahi and M. Egerstedt. Graph theoretic methods in multiagent networks. Princeton University Press, 2010.

[71] R. Goebel, R. G. Sanfelice, and A. R. Teel. Hybrid Dynamical Systems: Modeling, stability, and robustness. Princeton University Press, 2012.

[72] G. A. D. Lopes, B. Kersbergen, B. De Schutter, T. van den Boom, and R. Babuška. Synchronization of a class of cyclic discrete-event systems describing legged locomotion. Discrete Event Dynamic Systems, 26(2):225-261, 2016.

[73] S. Jiménez-Leudo, P. Nanez, N. Quijano, and C. F. Rodríguez. Juggling robots network-hybrid model and multi-agent synchronization. Master's thesis, Uniandes, 2016.

[74] X. Deng and Y. Yang. Communication synchronization in cluster-based sensor networks for cyber-physical systems. Transactions on Emerging Topics in Computing, 1(1):98-110, 2013.

[75] D. V. Dimarogonas, E. Frazzoli, and K. H. Johansson. Distributed event-triggered control for multi-agent systems. IEEE Transactions on Automatic Control, 57(5):1291-1297, 2012.

[76] Y. Fan, G. Feng, Y. Wang, and C. Song. Distributed eventtriggered control of multi-agent systems with combinational measurements. Automatica, 49(2):671-675, 2013.

[77] D. V. Dimarogonas, E. Frazzoli, and K. H. Johansson. Distributed self-triggered control for multi-agent systems. In 49th IEEE Conference on Decision and Control (CDC), pages 6716-6721. IEEE, 2010

[78] S. Phillips and R. G. Sanfelice. Robust distributed synchronization of networked linear systems with intermittent information. Automatica, 105:323-333, 2019.

[79] J. M. Hendrickx and J. N. Tsitsiklis. A new condition for convergence in continuous-time consensus seeking systems. In Proc. 50th. IEEE Conf. on Decision Control and 15th. Europ. Contr. Conf., pages 5070-5075, 2011.

[80] H. Su, Z. Rong, M. Z. Q. Chen, X. Wang, G. Chen, and H. Wang. Decentralized adaptive pinning control for cluster synchronization of complex dynamical networks. IEEE Transactions on Cybernetics, 43(1):394-399, 2013.

[81] W. Lu, B. Liu, and T. Chen. Cluster synchronization in networks of coupled nonidentical dynamical systems. Chaos: An Interdisciplinary Journal of Nonlinear Science, 20(1):013120, 2010.

[82] W. Wu, W. Zhou, and T. Chen. Cluster synchronization of linearly coupled complex networks under pinning control. IEEE Transactions on Circuits and Systems I: Regular Papers, 56(4):829-839, 2009.

[83] S. Martin, I-C. Morărescu, and D. Nešić. New cut-balance conditions in networks of clusters. Automatica, 77:180 - 183, 2017.

[84] P. Kokotović, H. Khalil, and J. O'reilly. Singular perturbation methods in control: analysis and design. SIAM, 1999.

[85] J. Chow and P. Kokotovic. Time scale modeling of sparse dynamic networks. IEEE Transactions on Automatic Control, 30(8):714-722, 1985.

[86] R. G. Sanfelice and A. R. Teel. On singular perturbations due to fast actuators in hybrid control systems. Automatica, 47(4):692-701, 2011

[87] I. Malloci, J. Daafouz, and C. Iung. Stability and stabilization of two time scale switched systems in discrete time. IEEE Transactions on Automatic Control, 55(6):1434-1438, 2010.

[88] J. Ben Rejeb, I-C. Morărescu, and J. Daafouz. Control design with guaranteed cost for synchronization in networks of linear singularly perturbed systems. Automatica, 91:89-97, 2018.

[89] J.J.B. Biemond, R. Postoyan, W. P. Heemels, H. Maurice, and N. Van de Wouw. Incremental stability of hybrid dynamical systems. IEEE Transactions on Automatic Control, 63(12):4094-4109, 2018

[90] S. Phillips and R. G. Sanfelice. Robust distributed synchronization of networked linear systems with intermittent information. Automatica, 105:323 - 333, 2019.

[91] A. R. Teel. Asymptotic stability for hybrid systems via de- 
composition, dissipativity, and detectability. In Proceedings of the 49th IEEE Conference on Decision and Control (CDC), pages 7419-7424. IEEE, 2010.

[92] C. Prieur, A. R. Teel, and L. Zaccarian. Relaxed persistent flow/jump conditions for uniform global asymptotic stability. Transactions on Automatic Control, 59(10):2766-2771, 2014.

[93] P. Bernard and R. Sanfelice. Robust Observer Design for Hybrid Dynamical Systems with Linear Maps and Approximately Known Jump Times. working paper or preprint, October 2019.

[94] M. Maghenem, A. Bautista-Castillo, E. Nuño, A. Loría, and E. Panteley. Consensus of multi-agent systems with nonholonomic restrictions via Lyapunov's direct method. IEEE Control Systems Letters, 3(2):344-349, 2019. DOI: 10.1109/LCSYS.2018.2879043.

[95] D. M. Hustig-Schultz and R. G. Sanfelice. A robust hybrid heavy ball algorithm for optimization with high performance. In Proceedings of the 2019 American Control Conference (ACC), pages 151-156, July 2019.

[96] A. H. Brodtkorb, S. A. Værnø, A. R. Teel, A. J. Sørensen, and R. Skjetne. Hybrid controller concept for dynamic positioning of marine vessels with experimental results. Automatica, 93:489-497, 2018.

[97] R. Beerens, A. Bisoffi, L. Zaccarian, W.P.M.H. Heemels, H. Nijmeijer, and N. van de Wouw. Reset integral control for improved settling of pid-based motion systems with friction. Automatica, 107:483 - 492, 2019

[98] R.G. Sanfelice, M. J. Messina, S. E. Tuna, and A. R. Teel. Robust hybrid controllers for continuous-time systems with applications to obstacle avoidance and regulation to disconnected set of points. In Proceedings of the 2006 American Control Conference, pages 6-pp. IEEE, 2006.

[99] G. Casadei, A. Isidori, and L. Marconi. About disconnected topologies and synchronization of homogeneous nonlinear agents over switching networks. International Journal of Robust and Nonlinear Control, 28(3):901-917, 2018.

[100] J. I. Poveda and A. R. Teel. Hybrid mechanisms for robust synchronization and coordination of multi-agent networked sampled-data systems. Automatica, 99:41-53, 2019.

[101] M. Maghenem, E. Panteley, and A. Loría. Singular- perturbations-based analysis of synchronization in heterogeneous networks: A case-study. In Proceedings of the 55th Conference on Decision and Control (CDC), pages 2581-2586. IEEE, 2016.

[102] E. Biyık and M. Arcak. Area aggregation and time-scale modeling for sparse nonlinear networks. Systems \& Control Letters, 57(2):142-149, 2008.

[103] S. Prajna, A. Jadbabaie, and G. J. Pappas. A framework for worst-case and stochastic safety verification using barrier certificates. IEEE Transactions on Automatic Control, 52(8):1415-1428, 2007.

[104] A. D. Ames, X. Xu, J. W. Grizzle, and P. Tabuada. Control barrier function based quadratic programs for safety critical systems. IEEE Transactions on Automatic Control, 62(8):3861-3876, 2017

[105] S. Coogan and M. Arcak. A dissipativity approach to safety verification for interconnected systems. IEEE Transactions on Automatic Control, 60(6):1722-1727, 2015.

106] C. K. Verginis and D. V. Dimarogonas. Closed-form barrier functions for multi-agent ellipsoidal systems with uncertain lagrangian dynamics. IEEE Control Systems Letters, 3(3):727$732,2019$.

[107] D. V. Dimarogonas. Sufficient conditions for decentralized potential functions based controllers using canonical vector fields. IEEE Transactions on Automatic Control, 57(10):2621-2626, 2012 .

[108] L. Lindemann and D. V. Dimarogonas. Control barrier functions for multi-agent systems under conflicting local signal temporal logic tasks. IEEE Control Systems Letters, 3(3):757-762, 2019 .

[109] P. Jagtap, A. Swikir, and M. Zamani. Compositional construction of control barrier functions for interconnected control systems. In Proceedings of the 23rd International Conference on Hybrid Systems: Computation and Control, HSCC 20, New York, NY, USA, 2020. Association for Computing Machinery.

[110] M. Maghenem and R. G. Sanfelice. Sufficient conditions for forward invariance and contractivity in hybrid inclusions using barrier functions. arXiv preprint:1908.03980, 2019. 